http://jmscr.igmpublication.org/home/ ISSN (e)-2347-176x ISSN (p) 2455-0450 crossref DOI: https://dx.doi.org/10.18535/jmscr/v8i11.35

\title{
Study of Epidemiological, Clinical, Laboratory Profile and Outcomes of Acute Pyelonephritis in a Tertiary Care Hospital of North Andhra Pradesh
}

\author{
Authors \\ Dr Tati Hyma ${ }^{1}$, Dr YG Sundara Raju², Dr Marri Lakshmi Harika ${ }^{3}$, \\ Dr Gadipudi Mounika ${ }^{4}$ \\ ${ }^{1,3,4}$ Post Graduates, Department of General Medicine \\ ${ }^{2}$ Professor, Department of General Medicine \\ King George Hospital, Visakhapatnam, Andhra Pradesh, India
}

\begin{abstract}
Background: Acute pyelonephritis is a severe form of urinary tract infection with symptoms that range from mild discomfort to life threatening illness or death. Complications may result in chronic renal scarring and renal function impairment, leading to more significant morbidity in patients with a preexisting structural or functional renal defect.

Materials and Methods: This is a prospective observational study done for eight months in the Department of General Medicine, King George Hospital, Visakhapatnam, from January 2020 to August 2020. A total of 40 patients with a diagnosis of acute pyelonephritis were followed up for this study

Results: Out of the 40 patients, 26 were male, and 14 were female. Renal dysfunction at presentation was seen in 28 patients. Urine culture was positive in 11 patients, with E.coli being the most common organism isolated. Ultrasound abdomen was suggestive of pyelonephritis in only 32 patients.

Conclusion: Acute pyelonephritis causes high morbidity and mortality and may even lead to chronic renal dysfunction. The absence of a positive urine culture does not rule out acute pyelonephritis. In a patient with high clinical suspicion of pyelonephritis and ultrasound abdomen does not reveal any abnormalities, CT abdomen should be performed to rule out the disease.
\end{abstract}

\section{Introduction}

Acute pyelonephritis is a bacterial infection causing inflammation of the kidneys. It occurs commonly due to an ascending urinary tract infection spreading from the bladder to the kidneys and collecting systems or can be due to hematogenous spread. Common presenting symptoms of acute pyelonephritis are fever, loin pain, burning micturition, increased frequency of micturition, nausea, and vomiting.

Emphysematous pyelonephritis is a necrotizing infection of the kidney, and it is seen more frequently in people with diabetes with an overall mortality rate of $30-40 \%{ }^{2}$. CT abdomen is more sensitive than ultrasound to diagnose emphysematous pyelonephritis. Outcomes were found to be better in patients treated with a combined medical and surgical approach than those treated with antibiotics alone.

\section{Aims and Objectives}

To study the epidemiological factors, risk factors, causative organisms, biochemical and radiological abnormalities, complications, and treatment outcomes in patients with acute pyelonephritis. 


\section{Materials and Methods}

This is a prospective observational study done for eight months from January 2020 to August 2020 in the Department of General Medicine, King George Hospital. This study included patients diagnosed with acute pyelonephritis and confirmed either with an ultrasound abdomen or CT abdomen. Patients of both sexes above 18 years of age were included in the study. A total of 40 patients were included in the study after taking informed consent.

Clinical features and laboratory data at presentation, management, and outcomes were studied prospectively.

Following investigations were done for all the patients:

1. Complete blood counts with ESR

2. Renal and liver function tests

3. Serum electrolytes

4. Blood glucose

5. Erythrocyte sedimentation rate and $\mathrm{C}$ reactive protein

6. Viral markers for HIV, hepatitis B, and hepatitis $\mathrm{C}$ viruses

7. Complete urine examination

8. Urine culture and sensitivity

9. Ultrasound abdomen

10. CT abdomen

HbA1C was done in patients who were already known to be diabetics and also in patients with fasting and postprandial blood sugar levels in the diabetic range

\section{Inclusion Criteria}

All patients aged $>18$ years with a diagnosis of acute pyelonephritis confirmed by ultrasound or CT abdomen were included in the study.

\section{Exclusion Criteria}

Following patients were excluded from the study:

1. History of kidney transplant

2. Pregnant women

3. Postoperative period of major urological surgery

\section{Management}

All the patients were treated with empirical antibiotics and then changed as per culture and antibiotic sensitivity reports. All patients received parenteral antibiotics for atleast one week, followed by oral antibiotics for two more weeks. Percutaneous nephrostomy was done for four patients with emphysematous pyelonephritis and a patient with renal abscess.

\section{Results}

Out of the 40 patients, 26 (65\%) were male and 14 $(35 \%)$ were female with a male to female ratio of $1.85: 1$.

$27(67.5 \%)$ patients were diabetics and $13(32.5 \%)$ patients were non diabetics. $13(32.5 \%)$ patients had either renal or ureteric calculi. $7(17.5 \%)$ patients had emphysematous pyelonephritis and $1(2.5 \%)$ patient had renal abscess. Bilateral involvement was seen in 6(15\%) patients. Renal dysfunction at presentation with a serum creatinine $>2 \mathrm{mg} / \mathrm{dl}$ was seen in $28(70 \%)$ patients.

Among the 40 patients, 32(80\%) had features suggestive of pyelonephritis on ultrasound abdomen and the remaining cases were confirmed with CT abdomen.

Urine culture was positive in only $11(27.5 \%)$ patients with E.coli being the most common organism which was isolated in 4 patients, whereas Klebsiella was isolated in 3 patients and pseudomonas aeruginosa isolated in 1 patient. 


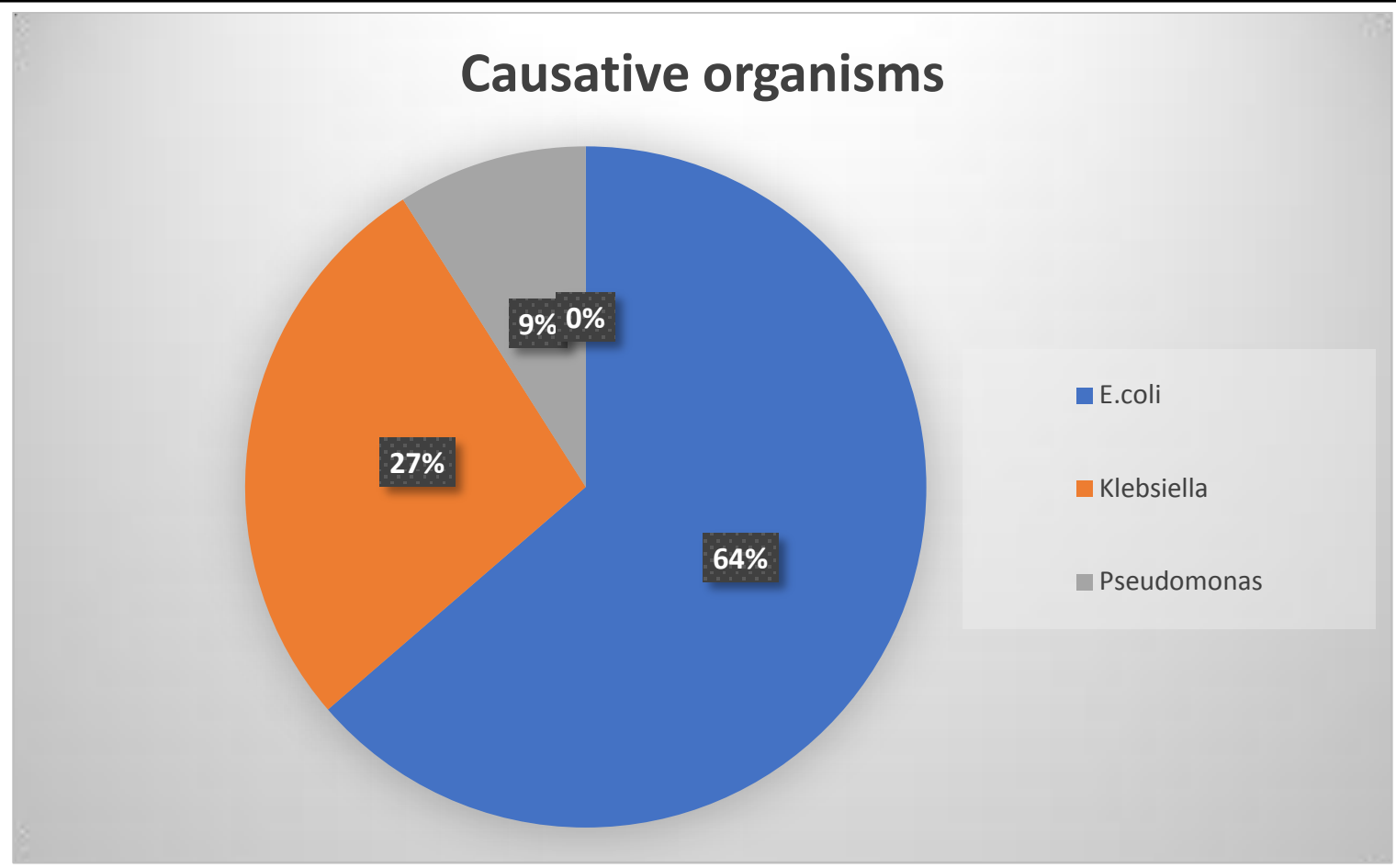

Among the diabetic patients, HbA1C was $>10 \%$ in $19(70.3 \%)$ patients, and it was more common in patients with emphysematous pyelonephritis.

Out of 40 patients, seven patients developed multi organ dysfunction syndrome and 4 out of them expired.

\section{Discussion}

In this study, the incidence of acute pyelonephritis is more common in males (65\%) than in females, which was similar to a study done by L. Umesha et al where $65.9 \%$ of the patients were male ${ }^{3}$.

Only 11 out of the 40 patients $(27.5 \%)$ had a positive urine culture in this study, which is similar to a study done by Georgi Abhiram et $\mathrm{al}^{4}$ where urine culture is positive in only $23.5 \%$. But in contrast to this study, positive urine culture was found in $48.3 \%$ patients in the study done by L.Umesha et $\mathrm{al}^{3}$.

Most common causative organism isolated was E.coli (63.6\%) which is similar to the studies done by Kumar $\mathrm{S}$ et $\mathrm{al}^{5}$, Patel $\mathrm{HB}$ et $\mathrm{al}^{6}$ and $\mathrm{K}$ Ramakrishnan et $\mathrm{al}^{7}$.

\section{Conclusion}

There is an increase in the incidence of acute pyelonephritis in recent times but there is no strong literature regarding the risk factors and causative organisms of this condition in Indian scenario. Acute pyelonephritis appears to be more common in diabetics than non-diabetics. High index of suspicion is needed in patients presenting with fever, loin pain, dysuria and even if ultrasound abdomen doesn't show any abnormality CT abdomen should be performed to diagnose acute pyelonephritis. Urine culture was positive in only $27.5 \%$ of the patients and in our study this was mostly attributed to prior antibiotic usage or late collection of samples. It is essential to diagnose it early and prompt antibiotic treatment started to prevent further renal damage which contributes highly to the mortality and morbidity. Local patterns of causative organisms and antibiotic sensitivity should be studied to formulate institutional antibiotic guidelines.

\section{Limitations}

The study is done in a limited number of patients. Results may vary when done in a large number of subjects.

Source of Support: None

Conflict of Interest: None 


\section{References}

1. Czaja CA, Scholes D, Hooton TM, Stamm WE: Population-based epidemiologic analysis of acute pyelonephritis. Clin Infect Dis. 2007, 45:273-280. 10. 1086/519268

2. Stone SC, Mallon WK, Childs JM, Docherty SD. Emphysematous pyelonephritis: clues to rapid diagnosis in the Emergency Department. J Emerg Med. 2005 Apr;28(3):315-9.

3. Umesha L, Shivaprasad SM, Rajiv EN, et al. Acute Pyelonephritis: A Single-center Experience. Indian J Nephrol. 2018;28(6): 454-461. doi:10.4103/ijn.IJN_219_16

4. Georgi Abraham, Yuvaram N.V. Reddy, Gautam George. Nephrology Dialysis Transplantation, Volume 27, Issue 9, September 2012, Pages 3391-3394.

5. Kumar S, Ramachandran R, Mete U, et al. Acute pyelonephritis in diabetes mellitus: single center experience. Indian J Nephrol. 2014;24:367-71

6. Patel HB, Soni ST, Bhagyalaxmi A, Patel NM. Causative agents of urinary tract infections and their antimicrobial susceptibility patterns at a referral center in Western India: An audit to help clinicians prevent antibiotic misuse. J Family Med Prim Care. 2019;8(1):154-159. doi:10.4103/jfmpc.jfmpc_203_18

7. Kalyanakrishnan Ramakrishnan, M.D., and Dewey C. Scheid, M.D., M.P.H., University of Oklahoma Health Sciences Center, Oklahoma City, Oklahoma Am Fam Physician. 2005 Mar 1;71(5):933-942. 